Article

\title{
Synthesis of Solution-Stable PEDOT-Coated Sulfonated Polystyrene Copolymer PEDOT:P(SS-co-St) Particles for All-Organic NIR-Shielding Films
}

\author{
Soeun Im, Chanil Park, Wonseok Cho, Jooyoung Kim, Minseok Jeong and Jung Hyun Kim * \\ Department of Chemical and Biomolecular Engineering, Yonsei University, 50 Yonsei-ro, Seodaemoon-Gu, \\ Seoul 03722, Korea; imse218@yonsei.ac.kr (S.I.); zzanil@nate.com (C.P.); intersv88@gmail.com (W.C.); \\ eyesmile21@naver.com (J.K.); cyberle2@naver.com (M.J.) \\ * Correspondence: jayhkim@yonsei.ac.kr; Tel.: +82-2-2123-7633
}

Received: 18 December 2018; Accepted: 22 February 2019; Published: 26 February 2019

check for updates

\begin{abstract}
We prepared poly(3,4-ethylenedioxythiophene) (PEDOT)-coated sulfonated polystyrene copolymer particles as efficient heat-shielding agents, which showed strong near-infrared (NIR) absorption, with high solid contents and good solution stability. The poly(styrene sulfonate-co-styrene) (P(SS-co-St)) copolymers were successfully synthesized via radical solution polymerization, and PEDOT-coated P(SS-co-St) (PEDOT:P(SS-co-St)) was synthesized via $\mathrm{Fe}^{+}$-catalyzed oxidative polymerization. PEDOT:P(SS-co-St) was characterized by nuclear magnetic resonance and Fourier transform infrared spectroscopies. The particle size and morphology of PEDOT:P(SS-co-St) were examined using transmission electron microscopy, dynamic light scattering, and zeta potential measurements. The maximum NIR-shielding efficiency of the film was $92.0 \%$ with $40 \%$ transmittance. The high solution stability of PEDOT:P(SS-co-St) make it an ideal candidate for heat-insulating materials that find application in semi-transparent heat-insulator-coated windows.
\end{abstract}

Keywords: conducting polymer; poly(3,4-ethylenedioxythiophene); PEDOT; core-shell particles; polystyrene; NIR shielding film

\section{Introduction}

Energy crisis is one of the most urgent problems threatening the society, and extensive effort is being devoted to improve energy efficiency and the responsible use of energy resources. Therefore, recent research has focused on the application of green technology in the fields of architecture and transportation to address these concerns. Semi-transparent heat insulation coatings are an essential next-generation green technology. Importantly, semi-transparent heat-insulator-coated windows serve distinct roles under different conditions. Transparent windows that are commonly used in buildings and transportation readily permit bi-directional heat flow. The energy distribution in solar irradiation consists of $5 \%$ in the ultraviolet range (UV; $280-400 \mathrm{~nm}$ ), $45 \%$ in the visible range $(400-780 \mathrm{~nm}$ ), and $50 \%$ in the near-infrared range (NIR; 780-2700 nm). Heat-shielding windows can not only block most of the NIR light from passing into a structure and keep it cool during summer, but also reduce heat loss during winter. NIR protection is required in many applications, as NIR light causes thermal aging and can lead to adverse health effects in humans [1,2]. Thus, the development of infrared-shielding materials has implications in energy use reduction and human health.

Heat-insulating materials usually block heat transfer by absorbing or reflecting infrared (IR) light. As NIR-shielding materials, conducting polymers (CPs), including conjugated polythiophene (PT), polyaniline (PANI) [3], and polypyrrole (PPy) [4], have attracted significant attention in 
recent years. Compared with some transparent thermal insulation coatings based on metals (such as $\mathrm{Ni}, \mathrm{Ag}$, and $\mathrm{Au}$ ), semiconducting oxides (such as tin-doped indium oxide (ITO) and antimony-doped tin oxide (ATO)) [5,6], and tungsten oxides $\left(\mathrm{M}_{X} \mathrm{WO}_{3}\right)$ [7], $\mathrm{CPs}$ are more promising for commercialization as NIR-shielding films due to their excellent processability, high flexibility, and low cost. CPs are frequently used in optoelectronic devices, including light emitting diodes (LEDs), solar cells, and touch panel displays, as they are relatively inexpensive, lightweight, and easily processable [8-10]. The unique properties of CPs implicate them in a nearly unlimited range of potential applications. CPs have long been studied in electrical and electronic applications for their ease of manufacture and excellent physical and electrical properties [11-13]. Among them, CPs, especially poly(3,4-ethylenedioxythiophene):poly(styrene sulfonate) (PEDOT:PSS) has attracted a great deal of attention in various applications such as transparent electrodes, solar cells, light-emitting diodes, and thermoelectric devices for reducing the processing cost or replacing expensive oxides.

In this paper, we report the synthesis of PEDOT:P(SS-co-St) (PEDOT = poly(3,4-ethylenedioxythiophene)) with a 50:50 molar ratio of poly(styrene sulfonate) to styrene for use as a metal-free NIR-shielding material. The hydrophobic styrene was first incorporated into the sodium salt of 4-styrenesulfonic acid (NaSS), yielding the amphiphilic copolymer P(SS-co-St). P(SS-co-St) can be easily polymerized via its vinyl functional groups, as well as processed as a monomer with styrene via radical polymerization and emulsifier-free copolymerization $[14,15]$. The amphiphilic copolymer P(SS-co-St) was readily synthesized via polymerization in aqueous solution. Then, the obtained P(SS-co-St) copolymer was used as a polymeric template in the oxidative polymerization of 3,4-ethylenedioxythiophene (EDOT), and the conductive dispersion product PEDOT:P(SS-co-St) exhibited colloidal stability and a high solid content. Furthermore, the heat-shielding properties of PEDOT:P(SS-co-St) composite films were evaluated. The maximum NIR-shielding efficiency of the film was $92.0 \%$ with $40 \%$ transmittance.

\section{Materials and Methods}

\subsection{Materials for P(SS-co-St) and PEDOT:P(SS-co-St) Synthesis}

4-Styrenesulfonic acid sodium salt hydrate (NaSS), styrene (St; 99\%) and 3,4-ethylenedioxythiophene (EDOT; 97\%) were purchased from Sigma-Aldrich (St. Louis, MO, USA) and were used without further purification. Sodium persulfate (NaPS) and iron(III) sulfate pentahydrate $\left(\mathrm{Fe}_{2}\left(\mathrm{SO}_{4}\right)_{3} \cdot 5 \mathrm{H}_{2} \mathrm{O}\right)$ were obtained from Sigma-Aldrich and used without further purification as reaction initiators. Double-distilled deionized water was used in all experiments.

\subsection{Preparation of $P(S S-c o-S t)$ Copolymers}

In the first step, a series of P(SS-co-St) copolymers containing one styrene per polymer chain were prepared via radical copolymerization in the presence of the water-soluble initiator NaPS. The styrene content in P(SS-co-St) was controlled by adding $50 \mathrm{~mol} \%$ styrene to the poly(styrenesulfonate) (PSS) polymers. Polymerization was performed in a four-necked double-jacketed glass reactor $(150 \mathrm{~mL})$ equipped with a mechanical stirrer, and was carried out at $80^{\circ} \mathrm{C}$ for $12 \mathrm{~h}$. In the reactor, NaSS was dissolved in $100 \mathrm{~mL}$ of ultra-pure water (UPW) and stirred at $250 \mathrm{rpm}$. The initiator solution was prepared by dissolving $0.065 \mathrm{~g}$ NaPS in $2 \mathrm{~mL}$ UPW, and was added to the reaction vessel within $15 \mathrm{~min}$. After the reaction period, the solution-phase copolymer product was ion-exchanged by mixing it with $31 \mathrm{~g}$ of positive ion exchangers for $1 \mathrm{~h}$ at room temperature to remove unreacted initiator, and was then filtered through a $30 \mu \mathrm{m}$ mesh.

\subsection{Synthesis of PEDOT:P(SS-co-St)}

The reactor was purged with nitrogen gas $\left(\mathrm{N}_{2} ; 99.999 \%\right)$ and mixed at $300 \mathrm{rpm}$. The initiators, $0.772 \mathrm{~g}$ NaPS dissolved in $2 \mathrm{~mL}$ UPW and $0.0053 \mathrm{~g} \mathrm{Fe} 2\left(\mathrm{SO}_{4}\right)_{3}$ dissolved in $3 \mathrm{~mL} \mathrm{UPW}$, were added to the reactants. After adding the EDOT monomer, the color of the solution gradually changed from yellow transparent to dark blue. The reaction was allowed to proceed for $24 \mathrm{~h}$ at $15^{\circ} \mathrm{C}$, with stirring 
at $300 \mathrm{rpm}$. After approximately $24 \mathrm{~h}$ of polymerization, PEDOT:P(SS-co-St) was purified via ion exchange by mixing the solution with positive and negative ion exchangers for $2 \mathrm{~h}$ at room temperature, followed by filtering it through a $30 \mu \mathrm{m}$ mesh.

\subsection{Characterization of P(SS-co-St) and PEDOT:P(SS-co-St)}

The chemical structures of PSS and $\mathrm{P}(\mathrm{SS}-\mathrm{co}-\mathrm{St})$ were analyzed by ${ }^{1} \mathrm{H}$ nuclear magnetic resonance (NMR) spectroscopy (Biospin Avance II, Bruker, Billerica, MA, USA) at $400 \mathrm{MHz}$ and by Fourier-transform infrared spectroscopy (FT-IR) (Vortex 70, Bruker) over the wavenumber range of $2000-400 \mathrm{~cm}^{-1}$ and with a resolution of $2 \mathrm{~cm}^{-1}$. The samples used for FT-IR and NMR were dried at $60{ }^{\circ} \mathrm{C}$ in a vacuum oven. NMR analysis was performed on solutions of PSS and P(SS-co-St) diluted in DMSO-d6. FT-IR analysis was performed using PSS and P(SS-co-St) in powder form mixed with $\mathrm{KBr}$ powder and palletized. The FT-IR absorption spectrum was recorded in transmittance mode.

The particle size and size distribution were measured using dynamic laser scattering (DLS; Zetasizer Nano S, Malvern Instruments, Worcestershire, UK). The morphology of the particles was observed using transmission electron microscopy (TEM; JSM 100CXII, UHR, JEOL, Tokyo, Japan). The NIR-shielding films were prepared by first filtering PEDOT:P(SS-co-St) through a nylon syringe filter $(5.0 \mu \mathrm{m})$ to remove impurities. The purified PEDOT:P(SS-co-St) solution was then directly coated on A4-sized PET substrates using the Mayer rod coating technique. First, the PEDOT:P(SS-co-St) solution was dropped onto the upper surface of the substrate, and the Mayer rod was quickly rolled over the PEDOT:P(SS-co-St) solution. Finally, the wet film of PEDOT:P(SS-co-St) spread over the surface of the substrate was dried at $150{ }^{\circ} \mathrm{C}$ for $2 \mathrm{~min}$ in a convection oven. Transmittance at $550 \mathrm{~nm}$ and absorption between 350 and $2000 \mathrm{~nm}$ were measured using a UV-VIS-NIR spectrophotometer (V-770, JASCO Corporation, Tokyo, Japan). Infrared bulbs ( $250 \mathrm{~W}$, Philips, Amsterdam, The Netherlands), black boxes, and thermometers were used to obtain temperature variation curves for the heat-shielding films. Briefly, a box measuring $300 \mathrm{~mm} \times 210 \mathrm{~mm} \times 150 \mathrm{~mm}$ made of black acrylate was prepared, and a thermometer was placed in the black box and used to measure variations in temperature. NIR-shielding film was used to cover a glass window on the upper side of the box. The distance between the black box and the infrared lamp was $500 \mathrm{~mm}$.

\section{Results and Discussion}

\subsection{Synthesis and Characterization of P(SS-co-St) Copolymers}

The hydrophobic styrene monomer influenced the water-solubility of PSS polymers in the continuous phase. The solution of the synthesized polymer with added styrene was translucent, and the originally hydrophilic polymer became amphiphilic upon addition of the styrene. The solubility of the copolymers in water controlled the core-shell morphology, via the introduction of hydrophobic styrene and hydrophilic NaSS monomers. Furthermore, the ${ }^{1} \mathrm{H}$ NMR spectra of the PSS and P(SS-co-St) (Figure 1) were analyzed in order to determine the copolymerization mechanism. The aromatic region of the spectra, along with the polymer structures (inset) and the peak assignments, indicate that peak $b$ (Figure 1) corresponds to the styrene in the copolymer $[16,17]$. The effect of styrene incorporation into the PSS polymer backbone is, thus, clearly exhibited in the NMR spectra. Analysis of the relative peak intensities of the NMR spectra of the polymers also provides information on the reactivity of the monomers during copolymerization. 

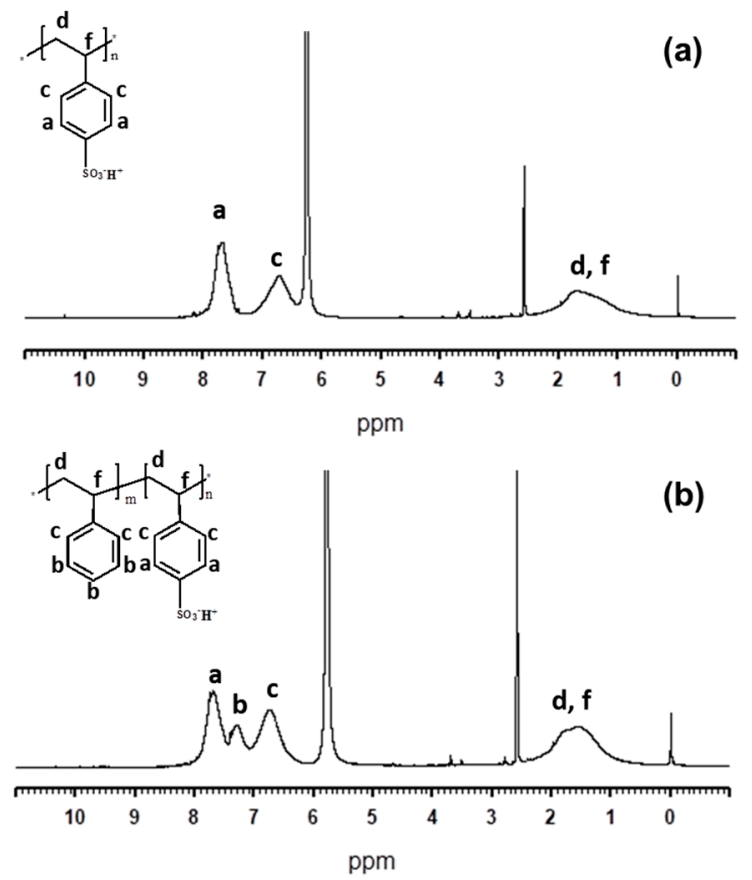

Figure 1. ${ }^{1} \mathrm{H}$ NMR spectra of (a) PSS and (b) P(SS-co-St).

A comparison of the FT-IR spectra of the PSS and P(SS-co-St) polymers is shown in Figure 2. The peaks in the $2800-3100 \mathrm{~cm}^{-1}$ region represent various vibration bands of polystyrene. The characteristic vibration bands of polystyrene are present in the spectrum of the P(SS-co-St) copolymer [16]. In addition, a previous study has reported that benzene ring stretching modes are observed at $1491 \mathrm{~cm}^{-1}$, out-of-plane ring deformation vibrations appear at $698 \mathrm{~cm}^{-1}$, and that the peak at $750 \mathrm{~cm}^{-1}$ is characteristic of the out-of-plane bending vibration of $\mathrm{C}-\mathrm{H}$ groups in mono-substituted benzene rings [18]. The peaks at 698,750 , and $1491 \mathrm{~cm}^{-1}$ increased in intensity as the styrene was added to the polymerization. These observations provide evidence that the synthesized P(SS-co-St) copolymer contained polystyrene. Both the spectra of PSS and P(SS-co-St) exhibited vibration bands at $1650 \mathrm{~cm}^{-1}$, which were assigned to water bound to the sulfonic groups of PSS. The intensity of this peak was much lower in P(SS-co-St) compared to that in pristine PSS, implying a relatively lower amount of absorbed water in the copolymer. The peak at $1182 \mathrm{~cm}^{-1}$ was assigned to an asymmetric stretching vibration in $-\mathrm{SO}_{3}$ resulting from the sulfonic acid hydrates $\left(-\mathrm{SO}_{3}{ }^{-} \mathrm{H}_{3} \mathrm{O}^{+}\right)$[19]. However, the intensities of these two peaks 1650 and $1182 \mathrm{~cm}^{-1}$ decreased when St was added to the polymer. These aforementioned peaks were identifiable in the spectra of both PSS and P(SS-co-St), meaning that the polymers contained $-\mathrm{SO}_{3}$ groups with different ionization states.

The size and morphology of P(SS-co-St) and PEDOT:P(SS-co-St) particles were determined from TEM images (Figure $3 \mathrm{a}, \mathrm{b}$ ). P(SS-co-St) consisted of spherical particles with size ranging from 80 to $200 \mathrm{~nm}$. Figure $3 \mathrm{~b}$ shows a TEM image of PEDOT:P(SS-co-St) spheres; a uniform PEDOT layer was observed on the surface of the P(SS-co-St) spheres. We obtained PEDOT:P(SS-co-St) core-shell particles using sulfonated PS microspheres as templates in the reaction system. Compared with the particle size shown in Figure 3a $(200 \mathrm{~nm})$, the size of the spherical PEDOT:P(SS-co-St) particles increased to $2 \mu \mathrm{m}$, which is because the PEDOT polymerization occurred along the radial direction from the surfaces of the P(SS-co-St) particles. This shows that the sulfonated PS microspheres served as templates. As explained in a previous report [20], a PSS layer formed around the PS spheres when PS template particles were first sulfonated with concentrated sulfuric acid. This is because sulfonation increased the polarity of the surfaces of the PS spheres. Therefore, the surfaces of the sulfonated PS microspheres exhibited negative charge when dispersed in water. After the introduction of the EDOT monomer, the P(SS-co-St) spheres were surrounded by PEDOT ${ }^{+}$with $\mathrm{PSS}^{-}$. $\mathrm{PEDOT}^{+}$and the excess PSS then 
formed PEDOT:PSS around the PS spheres. Therefore, PEDOT:P(SS-co-St) core-shell microspheres were obtained as a PEDOT:PSS layer covered the PS template.

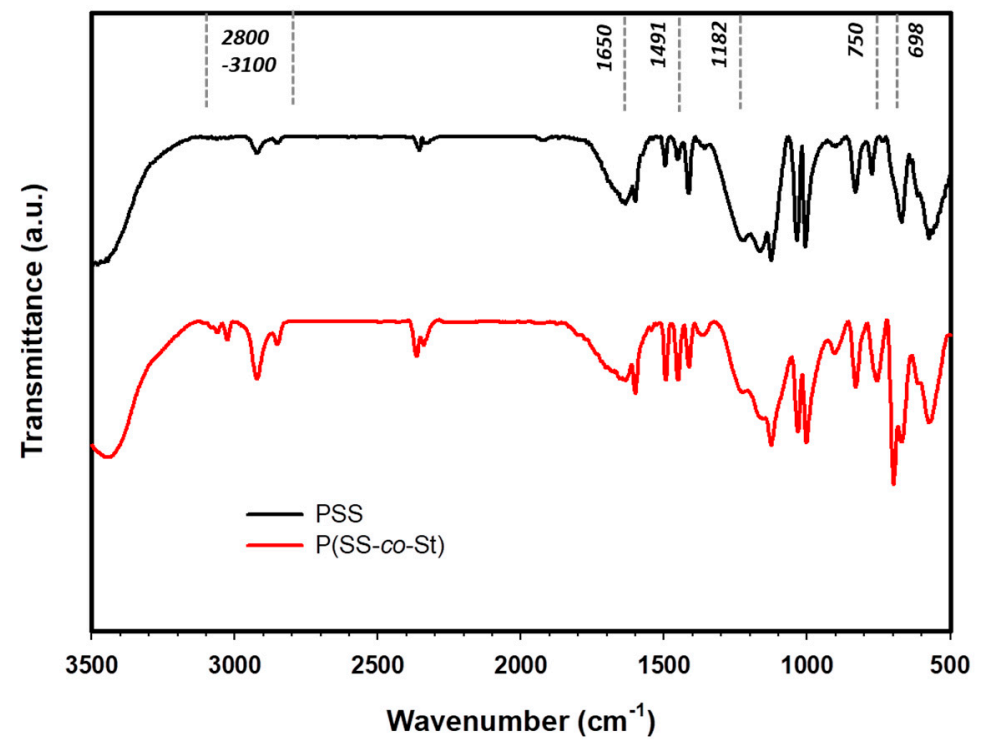

Figure 2. FT-IR spectra of PSS and P(SS-co-St) polymers.
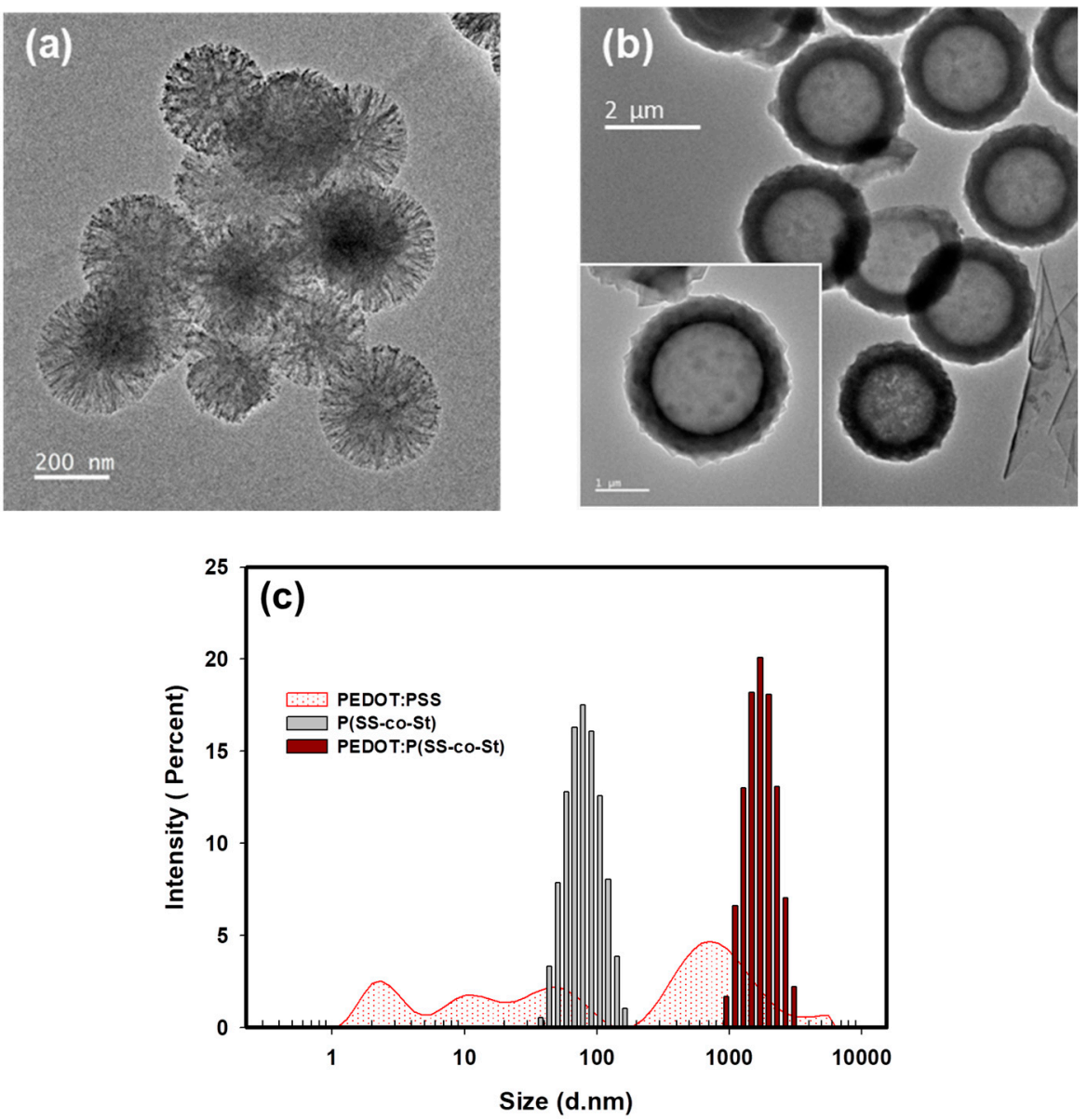

Figure 3. TEM images of (a) P(SS-co-St) and (b) PEDOT:P(SS-co-St); (c) DLS particle size distribution of PEDOT:PSS, P(SS-co-St), and PEDOT:P(SS-co-St). 
Figure $3 \mathrm{c}$ and Table 1 show the particle size and surface properties of the particles made of the various synthesized polymers in $\mathrm{H}_{2} \mathrm{O}$, as determined by DLS and zeta potential measurements [21]. The average diameter of P(SS-co-St) and PEDOT:P(SS-co-St) particles were 68.93 and $1695 \mathrm{~nm}$, respectively. Moreover, the $\mathrm{P}(\mathrm{SS}-\mathrm{co}-\mathrm{St})$ and PEDOT:P(SS-Co-St) particles exhibited zeta potentials of -74.9 and $-70.4 \mathrm{mV}$, respectively. These results indicate that the surfaces of the P(SS-co-St) and PEDOT:P(SS-co-St) particles were rich in sulfonyl groups [22]. On the other hand, PEDOT:PSS, with a solid content of $2 \%$, exhibited an irregular particle size distribution and zeta potential of $-40.4 \mathrm{mV}$, indicating relative instability in solution. The result of DLS measurements of PEDOT:PSS indicated primary nanoparticles with traces of clusters. PEDOT:PSS with a solid content of $2 \%$, which is higher than that of commercial PEDOT:PSS, can be predicted to exist in an unstable cohesive form in water due to its relatively low absolute zeta potential. This indicates that PEDOT:PSS with a $\%$ solid content would exist in an unstable form one month after being synthesized. The first step in synthesis of PEDOT:P(SS-co-St) composites involved preparation of a stable emulsion from surface-sulfonated PS particles by self-assembly of PS particles at the water-monomer interface. The hydrophilicity of styrene and NaSS was adjusted by controlling the composition of the particles; therefore, $-\mathrm{SO}_{3} \mathrm{H}$ groups on the particle surfaces act as stabilizers for the template particles when dispersed in water [23-25]. The entire preparation process is illustrated in Scheme 1.

Table 1. Z-average particle diameter, polydispersity index (PDI), and zeta potential for $\mathrm{P}(\mathrm{SS}-\mathrm{co}-\mathrm{St})$, PEDOT:P(SS-co-St), and PEDOT:PSS.

\begin{tabular}{cccc}
\hline Material & Z-average $\boldsymbol{d}(\mathbf{n m})$ & PDI & Zeta Potential $(\mathbf{m V})$ \\
\hline P(SS-co-St) & 68.93 & 0.236 & -74.9 \\
PEDOT:P(SS-co-St) & 1695 & 0.261 & -70.4 \\
PEDOT:PSS & 56.84 & 0.726 & -40.4 \\
\hline
\end{tabular}

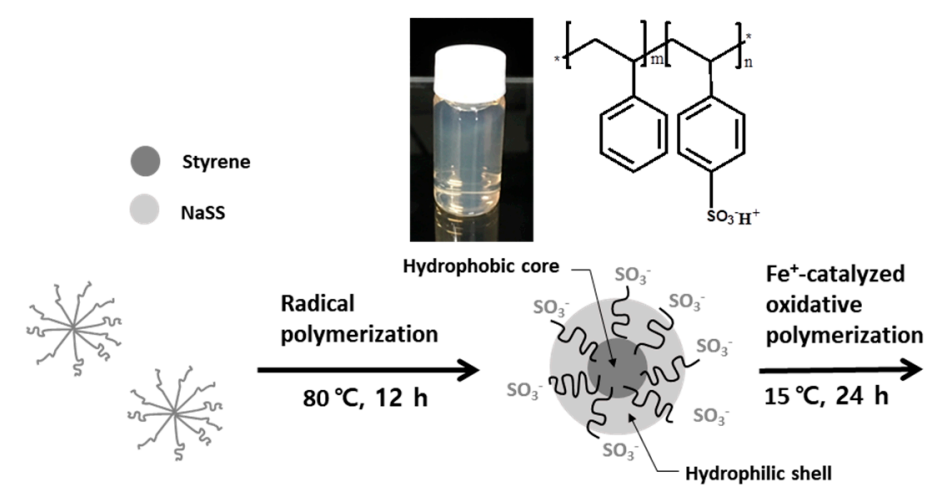

Swollen monomer micelle
P(SS-co-St) particle

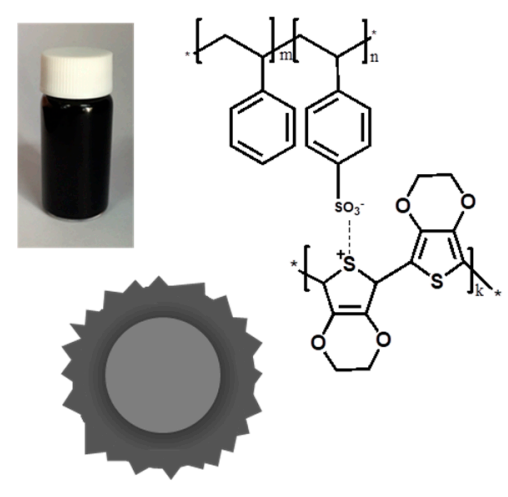

PEDOT:P(SS-co-St) particle

Scheme 1. Schematic of the formation of P(SS-co-St) and PEDOT:P(SS-co-St).

The relatively higher solid content in the aqueous PEDOT:PSS in this study decreased its solution stability, resulting in high viscosity and gelation. For this reason, the solid content in commercial PEDOT:PSS (PEDOT to PSS ratio of approximately 1:2.5) is approximately $1.3 \%$. The higher solid content requires an extremely well-controlled coating processes in order to guarantee high-quality film formation.

We synthesized CP dispersions under the same conditions with the PEDOT:PSS with a $2 \%$ solid content to compare the solution stabilities of PEDOT:PSS and PEDOT:P(SS-co-St). After the addition of $5 \%$ DMSO, the electrical conductivity of PEDOT:PSS was about $300 \mathrm{~S} / \mathrm{cm}$, whereas that of PEDOT:P(SS-co-St) was $250 \mathrm{~S} / \mathrm{cm}$, which is lower than that of the PEDOT:PSS. The high-solid-content PEDOT:PSS and PEDOT:P(SS-co-St) dispersions were stored for at least one month after synthesis, and the aged dispersions were then poured into Petri dishes to compare the stabilities of the 
dispersions; results are shown in Figure 4. The PEDOT:PSS dispersion partially gelated, while the PEDOT:P(SS-co-St) dispersion remained stable. The stability of PEDOT:P(SS-co-St) was, thus, remarkably enhanced due to its relatively high solid content $(2 \%)$.
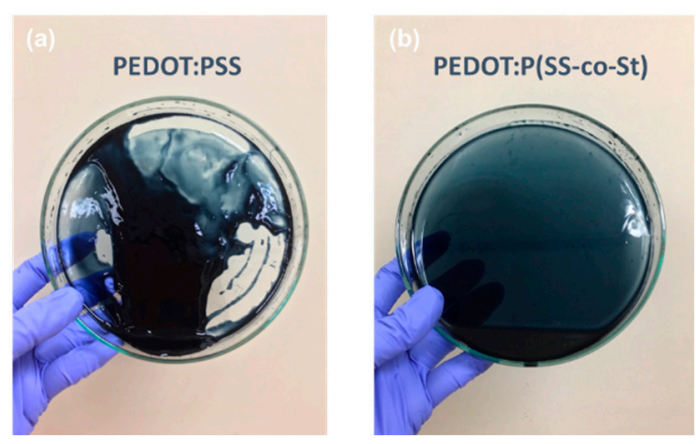

Figure 4. Solution-stability comparison of (a) pristine PEDOT:PSS and (b) PEDOT:P(SS-co-St).

\subsection{Heat-Shielding Efficiency of PEDOT:P(SS-co-St) NIR-Shielding Films}

NIR-shielding films composed of organic materials are cost-effective and have simple manufacturing processes. PEDOT has attracted much interest because of its high heat-shielding efficiency, great thermal stability, and high transparency when used in thin films [26,27]. However, like other conjugated polymers, PEDOT is insoluble in common organic solvents and water. In this study, this was resolved by using PSS as a charge-balancing dopant during polymerization of EDOT, and thus the resultant PEDOT:PSS dispersions were stable and had high conductivity [28]. However, PSS is a strong acid with a $\mathrm{pH}$ of less than 2, which adversely affects its lifetime and performance in many applications. Neutralization of PEDOT:PSS through the use of a base like $\mathrm{NaOH}$ is, thus, necessary to improve its lifetime and performance [27,29].

Yuta et al. suggested that the structure and electrical conductivity of PEDOT:PSS are dependent on $\mathrm{pH}$ [30]. Therefore, the NIR-shielding capability of an aqueous PEDOT:P(SS-co-St) dispersion depends on $\mathrm{pH}$. The optical properties of the aqueous dispersions were examined using UV-VIS-NIR spectroscopy after adjusting the $\mathrm{pH}$ with $1 \mathrm{M} \mathrm{NaOH}$ (Figure 5). The NIR-shielding effect of PEDOT:P(SS-Co-St) in solution was confirmed over a $\mathrm{pH}$ range from 2 to 5 ; there were no significant differences in the transmittance of the dispersions at different $\mathrm{pH}$ values. To determine the transmitted efficiency (TE) in the visible region (400-780 nm) and shielding efficiency (SE) in the NIR region $(780-2700 \mathrm{~nm})$, the standard solar spectrum is shown for comparison in Figures 5 and 6. In addition, the transmittance in the NIR region slightly increased, to almost the same extent as NIR shielding efficiency. This confirmed that the NIR-blocking capacity remained almost constant with increasing $\mathrm{pH}$. The PEDOT:P(SS-co-St) dispersion was, thus, considered suitable for use in practical applications.

The NIR-shielding efficiency of the PEDOT:P(SS-co-St) films with different transmittance profiles were also examined by UV-VIS-NIR measurements; the results are presented in Figure 6 and Table 2. We fabricated a heat-shielding film by varying the transmittance $(40 \%$ and $50 \%$ at $550 \mathrm{~nm})$ of the film, and confirmed the thermal efficiency of the films. The absorption at $789 \mathrm{~nm}$ is due to polaronic or bipolaronic absorption by the PEDOT shell [31,32]. As a result, the NIR-shielding effect of the PEDOT:P(SS-co-St) film depends on the film thickness. The thermal barrier effect improved as the transmittance at $550 \mathrm{~nm}$ was reduced from $50 \%$ to $40 \%$ and as the film thickness increased. In a previous study, we described the method for determining the shielding efficiency and presented the results [3]. Using this method, the NIR-shielding efficiency of the films were calculated to be $92.0 \%$ for the 40\%-transmittance film and $86.2 \%$ for the 50\%-transmittance film. Chen et al. previously reported that a polypyrrole-polyacrylic acid (PPy-PAA) film was able to block $92.1 \%$ of light in NIR region, but transmitted $32.1 \%$ of visible light [4]. The PEDOT:P(SS-co-St) films exhibited good transparency in the visible region and strong absorption in the NIR region. 


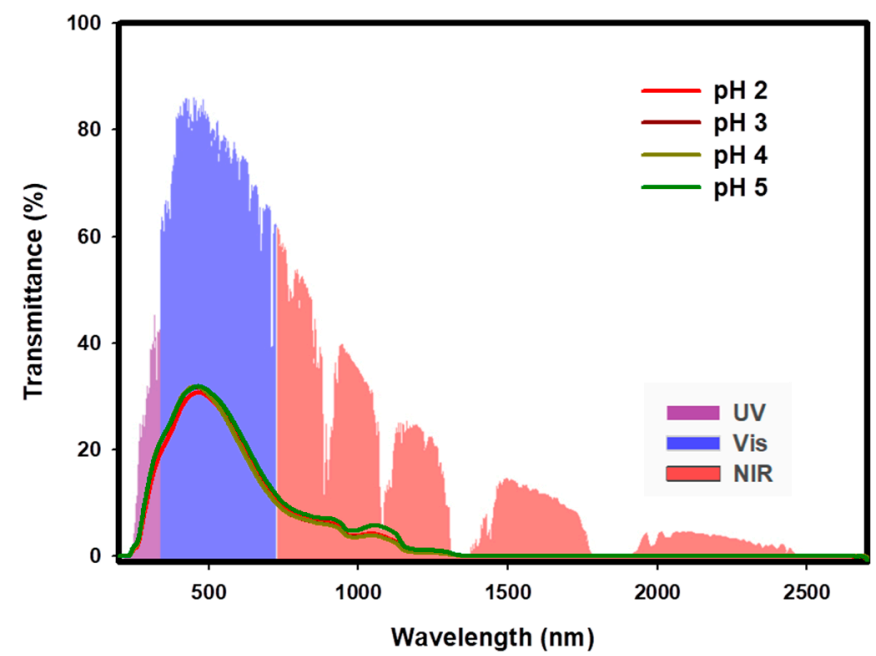

Figure 5. Transmittance spectra of PEDOT:P(SS-co-St) solutions of different $\mathrm{pH}$. The standard solar irradiation spectrum is provided for comparison.

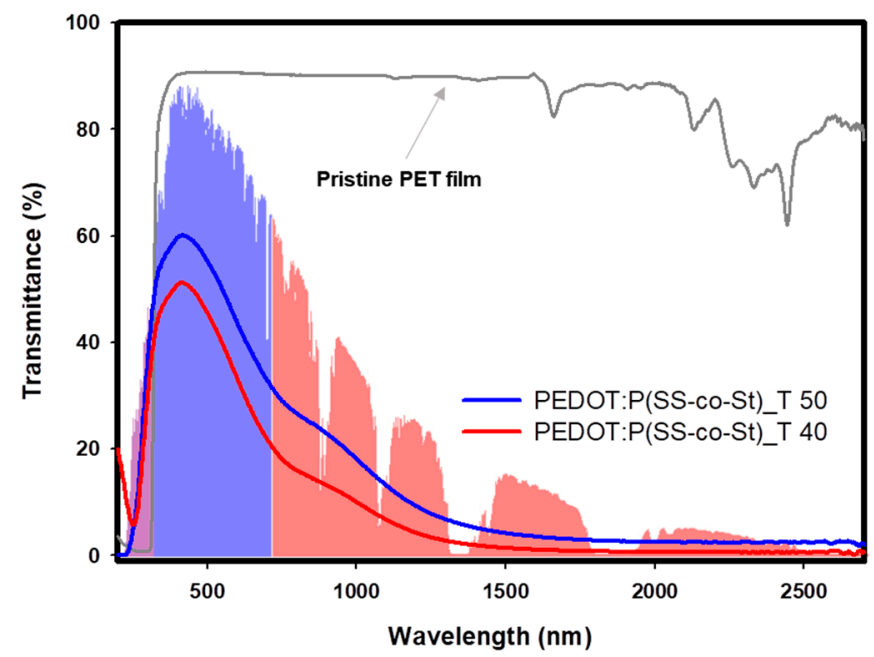

Figure 6. Transmittance spectra of PEDOT:P(SS-co-St) films with transmittance of $40 \%$ and $50 \%$ at $550 \mathrm{~nm}$. For comparison, the standard solar irradiation spectrum and the transmittance spectrum of the pristine PET film are provided.

Table 2. Transmittance in the visible region and shielding efficiency in the NIR region of selected PEDOT:P(SS-co-St) films.

\begin{tabular}{ccc}
\hline Sample & Transmittance (\%) $\mathbf{( 5 5 0} \mathbf{~ n m )}$ & $\begin{array}{c}\text { NIR Shielding Efficiency (\%) } \\
(\mathbf{7 8 0}-\mathbf{1 4 4 0} \mathbf{~ n m )}\end{array}$ \\
\hline T 50 & 50.05 & 86.25 \\
T 40 & 39.89 & 92.05 \\
\hline
\end{tabular}

Based on the aforementioned optical properties of the PEDOT:P(SS-co-St) films, it is reasonable to suggest that these films show great potential for application as NIR-shielding films. Figure 7 illustrates the thermal insulation experiment which was conducted, wherein a window of a sealed box was covered with bare PET or PEDOT:P(SS-co-St)-coated PET films and placed under an IR lamp. Figure $7 \mathrm{~b}$ shows the temperature variations inside the box after irradiation for $2000 \mathrm{~s}$, and indicates the overall variation between the initial and final temperatures. When the box was covered with a glass slide and a bare PET film was placed over the glass, the temperature increased significantly as 
irradiation time progressed $\left(\Delta T_{1}=20^{\circ} \mathrm{C}\right)$. The bare PET film thus did not provide any NIR shielding. This configuration was thus taken as a reference for comparison with the synthesized films. In contrast, the heating rate in the box covered with the glass slide and a PEDOT:P(SS-co-St) film was much lower than in the reference configuration. Further, the heating rate inside the box decreased distinctly as the film thickness was increased. After irradiation for $2000 \mathrm{~s}$, the overall temperature variation in the box covered with the PEDOT:P(SS-co-St) film with $50 \%$ transmittance ( 50 in Figure $7 \mathrm{~b} ; \Delta T_{2}=12{ }^{\circ} \mathrm{C}$ ), was lower than that for the reference configuration. Further, the temperature change in the box covered by the film with lower transmittance $(40 \%$; $\mathrm{T} 40)$ was even lower $\left(\Delta T_{3}=7^{\circ} \mathrm{C}\right)$. All PEDOT:P(SS-co-St) films were, thus, shown to provide good thermal insulation.

(a)

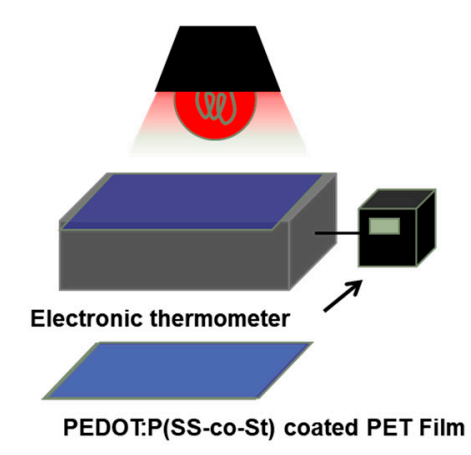

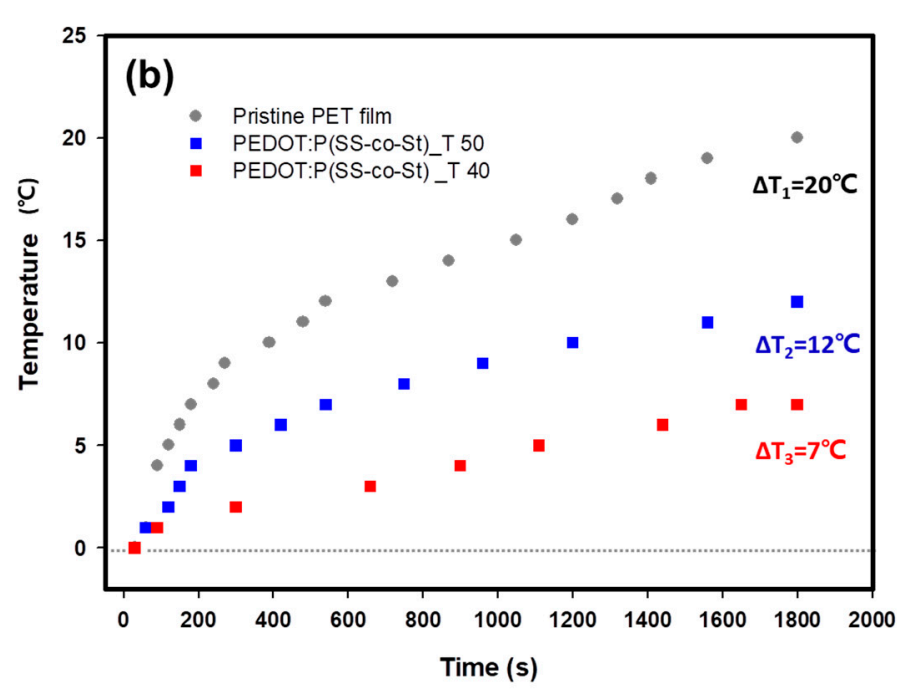

Figure 7. (a) Schematic illustration of the thermal insulation experiment, wherein a window in a sealed box was covered with a blank glass slide, commercial PET film, or PEDOT:P(SS-co-St)-coated PET Film. (b) Temperature changes of the air inside the box under the PET and PEDOT:P(SS-co-St)-coated PET films.

\section{Conclusions}

PEDOT:P(SS-co-St) dispersions were synthesized using a two-step process involving emulsifier-free emulsion copolymerization and oxidative polymerization. The PEDOT:P(SS-co-St) dispersions exhibited excellent multifunctional performance with high solid contents, stability, and NIR insulation capacity. The FT-IR and NMR results for PSS and P(SS-co-St) confirmed their successful polymerization. The size and morphology of the particles were determined via DLS measurements, zeta potential measurements, and inspection of TEM images. It was more difficult to stabilize the solution with higher solid content, which is an important factor in the coating process of $\mathrm{CP}$ films. A comparison between the synthesized copolymers and existing CPs showed that the limits of the existing CPs were exceeded by the polymers synthesized in this experiment. The UV-VIS-NIR measurements revealed the specific NIR-blocking capacity of the solutions as functions of $\mathrm{pH}$ and film thickness. A PEDOT:P(SS-co-St)-coated PET film was used as the window on a sealed black box and was illuminated with IR light. The interior air temperature increased to a smaller extent $\left(\Delta T_{2}\right.$ and $\Delta T_{3}=12$ and $7{ }^{\circ} \mathrm{C}$, respectively) than in the case wherein the window was covered with a bare PET film $\left(\Delta T_{1}=20^{\circ} \mathrm{C}\right)$. As a result of these properties, solution-stable PEDOT:P(SS-co-St) copolymers have great potential for use as semi-transparent heat insulation coatings.

Author Contributions: Conceptualization: W.C.; Data Curation: S.I.; Formal Analysis: S.I.; Investigation: C.P., J.K. and M.J.; Supervision: J.H.K.; Visualization: C.P.; Writing-Original Draft: S.I.

Funding: This research was supported by the Nano-Material Technology Development Program through the National Research Foundation of Korea (NRF) funded by the Ministry of Science, ICT and Future Planning (MSIP, 
Korea) (NRF-2014M3A7B4050960/2014M3A7B4051745). This work was further supported by the Technology Innovation Program (20002931) funded by the Ministry of Trade, Industry and Energy (MOTIE, Korea).

Conflicts of Interest: The authors declare no conflicts of interest.

\section{References}

1. Vierkötter, A.; Krutmann, J. Environmental influences on skin aging and ethnic-specific manifestations. Derm.-Endocrinol. 2012, 4, 227-231. [CrossRef] [PubMed]

2. Krutmann, J.; Morita, A.; Chung, J.H. Sun exposure: What molecular photodermatology tells us about its good and bad sides. J. Investig. Dermatol. 2012, 132, 976-984. [CrossRef] [PubMed]

3. Park, C.; Im, S.; Cho, W.; Kim, Y.; Kim, J.H. Facile synthesis of p (edot/ani): Pss with enhanced heat shielding efficiency via two-stage shot growth. RSC Adv. 2018, 8, 12992-12998. [CrossRef]

4. Chen, X.; Yu, N.; Zhang, L.; Liu, Z.; Wang, Z.; Chen, Z. Synthesis of polypyrrole nanoparticles for constructing full-polymer uv/nir-shielding film. RSC Adv. 2015, 5, 96888-96895. [CrossRef]

5. Matsui, H.; Hasebe, T.; Hasuike, N.; Tabata, H. Plasmonic heat shielding in the infrared range using oxide semiconductor nanoparticles based on sn-doped in2o3: Effect of size and interparticle gap. ACS Appl. Nano Mater. 2018, 1, 1853-1862. [CrossRef]

6. Miao, D.; Jiang, S.; Shang, S.; Chen, Z. Effect of heat treatment on infrared reflection property of al-doped zno films. Solar Energy Mater. Solar Cells 2014, 127, 163-168. [CrossRef]

7. Liu, T.; Liu, B.; Wang, J.; Yang, L.; Ma, X.; Li, H.; Zhang, Y.; Yin, S.; Sato, T.; Sekino, T. Smart window coating based on f-tio 2-k x wo 3 nanocomposites with heat shielding, ultraviolet isolating, hydrophilic and photocatalytic performance. Sci. Rep. 2016, 6, 27373. [CrossRef] [PubMed]

8. Im, S.; Kim, W.; Cho, W.; Shin, D.; Chun, D.H.; Rhee, R.; Kim, J.K.; Yi, Y.; Park, J.H.; Kim, J.H. Improved stability of interfacial energy-level alignment in inverted planar perovskite solar cells. ACS Appl. Mater. Interfaces 2018, 10, 18964-18973. [CrossRef] [PubMed]

9. Cho, W.; Hong, J.K.; Lee, J.J.; Kim, S.; Kim, S.; Im, S.; Yoo, D.; Kim, J.H. Synthesis of conductive and transparent pedot: $\mathrm{P}$ (ss-co-pegma) with excellent water-, weather-, and chemical-stabilities for organic solar cells. RSC Adv. 2016, 6, 63296-63303. [CrossRef]

10. Kim, W.; Kim, S.; Chai, S.U.; Jung, M.S.; Nam, J.K.; Kim, J.-H.; Park, J.H. Thermodynamically self-organized hole transport layers for high-efficiency inverted-planar perovskite solar cells. Nanoscale 2017, 9, 12677-12683. [CrossRef] [PubMed]

11. Kim, S.; Kim, S.Y.; Kim, J.; Kim, J.H. Highly reliable agnw/pedot: Pss hybrid films: Efficient methods for enhancing transparency and lowering resistance and haziness. J. Mater. Chem. C 2014, 2, 5636-5643. [CrossRef]

12. Cho, W.; Im, S.; Kim, S.; Kim, S.; Kim, J.H. Synthesis and characterization of pedot: P (ss-co-vtms) with hydrophobic properties and excellent thermal stability. Polymers 2016, 8, 189. [CrossRef]

13. Kim, Y.; Cho, W.; Kim, Y.; Cho, H.; Kim, J.H. Electrical characteristics of heterogeneous polymer layers in pedot: Pss films. J. Mater. Chem. C 2018, 6, 8906-8913. [CrossRef]

14. Kim, J.; Chainey, M.L.; El-Aasser, M.; Vanderhoff, J. Emulsifier-free emulsion copolymerization of styrene and sodium styrene sulfonate. J. Polym. Sci. Part A Polym. Chem. 1992, 30, 171-183. [CrossRef]

15. Sen, A.K.; Roy, S.; Juvekar, V.A. Effect of structure on solution and interfacial properties of sodium polystyrene sulfonate (napss). Polym. Int. 2007, 56, 167-174. [CrossRef]

16. Arunbabu, D.; Sanga, Z.; Seenimeera, K.M.; Jana, T. Emulsion copolymerization of styrene and sodium styrene sulfonate: Kinetics, monomer reactivity ratios and copolymer properties. Polym. Int. 2009, 58, 88-96. [CrossRef]

17. Lee, D.K.; Park, J.T.; Roh, D.K.; Min, B.R.; Kim, J.H. Synthesis of crosslinked polystyrene-b-poly (hydroxyethyl methacrylate)-b-poly (styrene sulfonic acid) triblock copolymer for electrolyte membranes. Macromol. Res. 2009, 17, 325-331. [CrossRef]

18. Kim, S.H.; Kim, J.H.; Choi, H.J.; Park, J. Pickering emulsion polymerized poly (3,4-ethylenedioxythiophene): Poly (styrenesulfonate)/polystyrene composite particles and their electric stimuli-response. RSC Adv. 2015, 5, 72387-72393. [CrossRef]

19. Brijmohan, S.B.; Swier, S.; Weiss, R.; Shaw, M.T. Synthesis and characterization of cross-linked sulfonated polystyrene nanoparticles. Ind. Eng. Chem. Res. 2005, 44, 8039-8045. [CrossRef] 
20. Zhu, C.; Chen, Z.; Ni, C.; Yu, J.; Huang, B.; Shan, M. Degradation of sodium polystyrene sulfonate and the radical initiated polymerization of styrene under ultrasonic irradiation. Polym.-Plast. Technol. Eng. 2011, 50, 1262-1265. [CrossRef]

21. Ryu, H.W.; Kim, Y.S.; Kim, J.H.; Cheong, I.W. Direct synthetic route for water-dispersible polythiophene nanoparticles via surfactant-free oxidative polymerization. Polymer 2014, 55, 806-812. [CrossRef]

22. Horikawa, M.; Fujiki, T.; Shirosaki, T.; Ryu, N.; Sakurai, H.; Nagaoka, S.; Ihara, H. The development of a highly conductive pedot system by doping with partially crystalline sulfated cellulose and its electric conductivity. J. Mater. Chem. C 2015, 3, 8881-8887. [CrossRef]

23. Yao, Q.; Wilkie, C.A. Thermal degradation of blends of polystyrene and poly (sodium 4-styrenesulfonate) and the copolymer, poly (styrene-co-sodium 4-styrenesulfonate). Polym. Degrad. Stab. 1999, 66, 379-384. [CrossRef]

24. Fan, X.; Niu, L.; Xia, Z. Preparation of raspberry-like silica microcapsules via sulfonated polystyrene template and aniline medium assembly method. Colloid Polym. Sci. 2014, 292, 3251-3259. [CrossRef]

25. Lee, S.M.; Lee, S.J.; Kim, J.H.; Cheong, I.W. Synthesis of polystyrene/polythiophene core/shell nanoparticles by dual initiation. Polymer 2011, 52, 4227-4234. [CrossRef]

26. Chang-Jian, C.W.; Cho, E.C.; Yen, S.C.; Ho, B.C.; Lee, K.C.; Huang, J.H.; Hsiao, Y.S. Facile preparation of $\mathrm{wo}_{3}$ / pedot: PSS composite for inkjet printed electrochromic window and its performance for heat shielding. Dyes Pigments 2018, 148, 465-473. [CrossRef]

27. Yoon, H.J.; Jeong, S.Y.; Lee, S.; jic Shin, G.; Choi, K.H. Near infrared shielding properties of pedot: PSS with different additives. In Proceedings of the 2012 4th International Conference on Chemical, Biological and Environmental Engineering, Phuket, Thailand, 1 September 2012.

28. Kim, J.; Jung, J.; Lee, D.; Joo, J. Enhancement of electrical conductivity of poly (3,4-ethylenedioxythiophene)/poly (4-styrenesulfonate) by a change of solvents. Synth. Metals 2002, 126, 311-316. [CrossRef]

29. Cho, A.; Kim, S.; Kim, S.; Cho, W.; Park, C.; Kim, F.S.; Kim, J.H. Influence of imidazole-based acidity control of pedot: PSS on its electrical properties and environmental stability. J. Polym. Sci. Part B Polym. Phys. 2016, 54, 1530-1536. [CrossRef]

30. Mochizuki, Y.; Horii, T.; Okuzaki, H. Effect of ph on structure and conductivity of pedot/pss. Trans. Mater. Res. Soc. Jpn. 2012, 37, 307-310. [CrossRef]

31. Han, M.G.; Foulger, S.H. Crystalline colloidal arrays composed of poly (3,4-ethylenedioxythiophene)-coated polystyrene particles with a stop band in the visible regime. Adv. Mater. 2004, 16, 231-234. [CrossRef]

32. Han, M.G.; Foulger, S.H. Preparation of poly (3,4-ethylenedioxythiophene)(pedot) coated silica core-shell particles and pedot hollow particles. Chem. Commun. 2004, 19, 2154-2155. [CrossRef] [PubMed] 